\section{AB0954 RELATIONSHIP BETWEEN SERUM COMPLEMENT LEVELS AND RENAL PATHOLOGICAL CLASSIFICATION IN CHILDREN WITH SILENT LUPUS NEPHRITIS}

H. Wakiguchi ${ }^{1}$, T. Kubota ${ }^{2}$, S. Takei ${ }^{3} \cdot{ }^{1}$ Department of Pediatrics, Yamaguchi University Graduate School of Medicine, Ube: ${ }^{2}$ Department of Pediatrics, Kagoshima University Graduate School of Medical and Dental Sciences; ${ }^{3}$ School of Health Science, Faculty of Medicine, Kagoshima University, Kagoshima, Japan

Background: Silent lupus nephritis is defined by some pathological evidence of lupus nephritis with normal urinary findings. It is therefore apparent only upon renal biopsy. Serum complement levels have been associated with overall lupus disease activity. Despite recent progress in understanding the pathogenesis of lupus nephritis, including complement, our understanding of silent lupus nephritis, especially in children, remains limited.

Objectives: To search whether serum complement levels are associated with renal pathological classification in children with silent lupus nephritis.

Methods: We determined serum C3 and C4 levels and International Society of Nephrology/Renal Pathology Society classification in 25 patients with paediatric silent lupus nephritis before initial therapy who were admitted to our hospital. Patients were classified as having silent lupus nephritis based on normal urinary findings at baseline renal biopsy in juvenile systemic lupus erythematosus.

Results: Serum C3 levels varied between International Society of Nephrology/Renal Pathology Society classes, with significantly lower levels for silent lupus nephritis patients in class III compared to class II, and for class II compared to class I. There was no significant difference in serum C4 levels between International Society of Nephrology/Renal Pathology Society classes in patients with silent lupus nephritis.

Conclusions: Our results suggest that serum C3 levels were associated with renal pathological classification in children with silent lupus nephritis. We propose that serum C3 levels would provide a useful tool for predicting latent severe nephritis in patients with juvenile systemic lupus erythematosus who have normal urinary findings before initial therapy.

Disclosure of Interest: None declared

DOI: 10.1136/annrheumdis-2017-eular.1012

\section{AB0955 NEW ONSET OF UVEITIS, PSORIASIS OR IBD AS PARADOXICAL EFFECTS OF BIOLOGICS IN PATIENTS WITH JUVENILE IDIOPATHIC ARTHRITIS: SINGLE CENTER EXPERIENCE}

I. Nikishina, O. Kostareva, M. Kaleda, S. Rodionovskaya, S. Arsenyeva, D. Alekseev, E. Fedorov, A. Shapovalenko. V.A.Nasonova Research Institute of Rheumatology, Moscow, Russian Federation

Background: Biological agents (BA) are high efficacy options for current therapy for patients (pts) with juvenile idiopathic arthritis (JIA). They are successfully used not only for the arthritis but also for uveitis, psoriasis and inflammatory bowel disease (IBD). However, paradoxical induction of these conditions in pts treated with BA is a well-established phenomenon.

Objectives: To evaluate the frequency of new onset of uveitis, psoriasis or IBD occurring under BA therapy in JIA pts, to establish clinical features, which may be associated with development of such effects.

Methods: Retrospective cohort study involved all JIA pts (740) who were treated with BA in our clinic from 2004 to 2016. All cases of new onset (no)-uveitis/psoriasis/IBD collected; clinical features of disease onset and course, activity level, JIA category, exposure to Methotrexate (MTX) and BA, presence of ANA, HLA B27 were studied.

Results: We identified $20(2.7 \%)$ pts (11 female/9 male) with no-uveitis under BA, mostly during etanercept (ETA) therapy (18 cases from 285 ETA courses, $6.3 \%), 1 / 112$ - in abatacept (ABA) and 1/233 - in adalimumab (ADA); 2.46 events/100 patient-year (PY) vs 0.31 in ABA, and 0.15 in ADA), ETA exposure was 14.9 \pm 9.9 months (mo). JIA subtypes were as follows: RF-neg polyarthritis 5 (25\%), persistent oligoarthritis $3(15 \%)$, extended oligoarthritis $10(50 \%)$ ERA - 2 $(10 \%)$. JIA started in this group at the age of $4.5 \pm 3.9 \mathrm{yrs} .18 / 20$ patients had high laboratory activity (CRP $55 \pm 25 \mathrm{mg} / \mathrm{l}$ ) and severe arthritis before BA initiation. Most of pts (16/20) achieved $90-100 \%$ ACRpedi-response by the uveitis development. 1 pt was treated by ADA for the $71 \mathrm{mo}$ and switched to ABA. in 1 pt no-uveitis was obseved under ABA. 12/20 pts were ANA-positive, 10/20 pts had HLAB27, $1 \mathrm{pt}$ did not have ANA or HLAB27. Uveitis was occurred earlier in ANA plus B27 positive pts (mean exposure - 10.7 mo vs 27.4 in ANA+ and 21.6 in B27+ pts). $17 / 20(85 \%)$ of pts received MTX. In all cases of no-uveitis BA was switched. 5 pts from $740(0.7 \%)$ developed no-psoriasis under BA: INF - 2 cases $(0.62 / 100 P Y)$, ADA - 2 (0.15/100PY), ABA -1 (0.31/100PY). JIA subtypes were presented by RF-negative polyarthritis (1), extended oligoarthritis -3 . All received MTX. 1/5 pts was ANA+, 2/5 - HLAB27+. Average age of disease onset was $9.8 \pm 7.8$ years; BA exposure before psoriasis was $25 \pm 11.5 \mathrm{mo}$. Therapy was continued in $3 / 5$ pts; switched from INF to ADA in 2 . Only 1 case of no-IBD was occurred in female patient fulfilled to systemic-onset JIA criteria years. She was treated by INF for the 62 months and switched to ADA due to secondary inefficiency presented by active polyarthritis and sacroilitis (HLAB27neg), but 2 months later severe gastrointestinal symptoms appeared and diagnosis of Crohn's disease was established by endoscopy.

Conclusions: Our study suggested that new onset of uveitis, psoriasis and IBD is rare adverse event during BA therapy in JiA. It seemes to be as delayed implication of disease natura. but not therapy complication. Uveitis observed mostly in pts receiving ETA, unlike the psoriasis mainly developed during TNF-monoclonal antibodies using. High activity aggressive manifestations at disease onset and good initial response to BA are typical features for all pts, who developed paradoxical effects under BA therapy.

Disclosure of Interest: None declared

DOI: 10.1136/annrheumdis-2017-eular.6753

\section{AB0956 INFLUENCE OF BIOLOGICAL THERAPY ON BONE MINERAL DENSITY IN CHILDREN WITH JUVENILE IDIOPATHIC ARTHRITIS}

I. Chyzheuskaya ${ }^{1}$, L. Byelyaeva ${ }^{2}$, N. Vasiljeva ${ }^{2}$, R. Filonovich ${ }^{1}$, L. Zajtseva ${ }^{1}$. ${ }^{1}$ Department of pediatrics, 4 th City Children's Clinical Hospital of Minsk; ${ }^{2}$ Department of pediatrics, Belarusian Medical Academy of Postgraduate Education, Minsk, Belarus

Background: Juvenile idiopathic arthritis (JIA) is one of the most common and most disabling rheumatic diseases in children. JIA is a systemic chronic inflammatory immunopathological disease, which leads to dysfunction of the joints, their deformation and limitations of life of the child, violates the child's growth and development. One of the complications of systemic JIA in children is osteoporosis. In the pathogenesis of osteoporosis important place is given to the use of glucocorticoids. In world Rheumatology successfully used drugs aimed at the major pro-inflammatory cytokines such as tumor necrosis factor (TNF), interleukin-1 (IL-1), interleukin-6 (IL-6), and others.

Objectives: The purpose of research - to determine the influence of biological therapy on bone mineral density in children with JIA.

Methods: 15 children with systemic JIA (mean age 11,9 $\pm 3,4$ years) were examined in the rheumatological department of 4 th city clinical hospital of Minsk. Bone mineral density was assessed by 2-energy $\mathrm{X}$-ray absorptiometry (DEXA) at two points of the skeleton. The level of mineralization of skeletal mineral content was estimated in the bone tissue (BMC), bone mineral density (BMD) and the parameter Z-score. Z-score was used to determine the incidence of osteopenia and osteoporosis in children surveyed. In accordance with the WHO criteria for normal bone mineral density was diagnosed with the Z-score $>-1$ SD, osteopenia at Z-score <-1 SD, but> - 2,5 SD, osteoporosis - with Z-score <-2,5 SD. Statistical data processing carried out using an integrated application package "Statistics 6 for Windows".

Results: In a study of children osteopenia was diagnosed in 9 patients with systemic JIA (mean Z-score -2,3 SD) Osteoporosis was diagnosed in 6 patients with systemic JIA (mean Z-score -2,7 SD). All children received a mean dose of methotrexate $12.7 \mathrm{mg} / \mathrm{m}^{2}$ of body surface area, an average dose of methylprednisolone $0.34 \mathrm{mg} / \mathrm{kg}$ body weight per day and treatment of IL-6 inhibitor - tocilizumab $8 \mathrm{mg} / \mathrm{kg}$ body weight every 2-4 weeks. Bone mineral density was measured prior to initiating therapy tocilizumab and after 2 years of therapy.

During tocilizumab therapy achieved remission of the disease, all children was canceled methylprednisolone. After re densitometry of 2 years after the beginning of therapy improvement noted tocilizumab Z-score in children with osteopenia with Z-score -2.3 SD to Z-score -1.4 SD, in children with osteoporosis a Z-score $-27 \mathrm{SD}$ to Z-score - 2,1 SD.

Conclusions: The results indicate a positive influence tocilizumab therapy to bone mineral density in children with JIA.

Acknowledgements: This study would not have been possible without the collaboration of numerous Belarusian pediatric rheumatologists, patients and their parents.

Disclosure of Interest: None declared

DOI: 10.1136/annrheumdis-2017-eular.6787

\section{AB0957 IS THERE A DIFFERENCE IN THE CLINICAL PRESENTATION OF JUVENILE SYSTEMIC SCLERODERMA PATIENTS ACCORDING THE AGE OF ONSET: RESULTS FROM THE JUVENILE SCLERODERMA INCEPTION COHORT WWW.JUVENILE-SCLERODERMA.COM}

I. Foeldvari ${ }^{1}$, J. Klotsche ${ }^{2}$, O. Kasapçopur ${ }^{1}$, A. Adrovic ${ }^{1}$, V. Stanevicha ${ }^{1}$ M.T. Terreri ${ }^{1}$, E. Alexeeva ${ }^{1}$, M. Katsicas ${ }^{1}$, V. Smith ${ }^{1}$, R. Cimaz ${ }^{1}$, M. Kostik ${ }^{1}$, T. Lehman ${ }^{1}$, J. Anton ${ }^{1}$, W.-A. Sifuentes-Giraldo ${ }^{1}$, F. Sztajnbok ${ }^{1}$, T. Avcin ${ }^{1}$, M. Janarthanan ${ }^{1}$, M.J. Santos ${ }^{1}$, M. Moll ${ }^{1}$, D. Nemcova ${ }^{1}$, C. Battagliotti ${ }^{1}$, J. Brunner ${ }^{1}$, D. Eleftheriou ${ }^{1}$, L. Harel ${ }^{1}$, T. Kallinich ${ }^{1}$, K. Minden ${ }^{2}$, S. Nielsen ${ }^{1}$, K. Torok ${ }^{1}$, Y. Uziel ${ }^{1}$, A. Stevens ${ }^{1}$, C. Pilkington ${ }^{1}$, N. Helmus ${ }^{1}$. ${ }^{1}$ Hamburg Center for Pediatric Rheumatology, Hamburg; ${ }^{2}$ DRFZ, Berlin, Germany

Background: Juvenile systemic sclerosis (jSSc) is an orphan autoimmune disease. It was rarely looked at the differences between the clinical presentations of patients at different paediatric age groups. The juvenile scleroderma inception cohort (www.juvenile-scleroderma.com) is a prospective standardized register for patients with jSSc.

Objectives: Comparison of clinical characteristics of patients with different age range at the time of inclusion in the registry.

Methods: Patients with jSSc were included worldwide to the juvenile scleroderma inception cohort. We compared the demographics and clinical characteristics of 